40 SNAP, CRACKLE \& POP: DEVELOPING A FRAMEWORK TO ASSESS AND SUPPORT NURSING STUDENTS DURING FULL TERM PLACEMENT WITH AN ACUTE HOSPITAL SPECIALIST PALLIATIVE CARE TEAM

Paul Spencer, Christopher Seymour. Manchester University NHS Foundation Trust

10.1136/spcare-2020-PCC.61

Background In 2019 our acute hospital specialist palliative care team became a full term placement option for nursing students. We aimed to develop a framework that assessed proficiencies, encouraged creative, reflective thinking and provided outcomes of learning for students during their time with us. Methods

We performed a curriculum mapping exercise of the new NMC Standards of proficiency alongside a literature review of teaching methodologies. Findings from these exercises were combined with a newer concept we developed called 'creative reflection' resulting in the SNaP, CRACKLE \& POP framework.

Results SNaP: Simulated Narrative Practice

Simulation based learning utilising scenarios pertinent to palliative practice to create a safe space, in which our students can receive constructive feedback at a controlled pace with opportunity to run scenarios again after they has been delivered.

CRACKLE: Creative Reflection Assessing Clinical Knowledge \& Learning Experience

Students are asked to present a reflective account to the Supportive and Palliative Care Team. We ask that this is done in a creative manner our only rule being no PowerPoint. CRACKLE enables our students to engage with their reflections on practice differently making it a shared and memorable experience.

POP: Proactive Online Practice

We ensure our students make use of the nationally recognised online learning resource e-ELCA.

Conclusion SNaP, CRACKLE \& POP is adaptable to the learning needs of the individual, offers a safe space to practice and reflect upon complex situations and teaches a range of key transferable skills. The framework has been successfully implemented with our first cohort of students and has proved to challenge perceptions of palliative care, the dying process and the roles and responsibilities of clinical nurse specialists. Feedback indicates this framework to be effective in assessing and supporting our nursing students during placement.

\section{RESEARCH EXPERIENCE, INTEREST \& OPPORTUNITIES: FINDINGS FROM A NATIONAL UK PALLIATIVE MEDICINE TRAINING SURVEY}

Donna Wakefield, Charlotte Chamberlain, Jonathan Koffman. UK Palliative Trainee Research Collaborative (UK_PRC)

\subsection{6/spcare-2020-PCC.62}

Background Palliative Medicine has rapidly grown but the volume of research currently remains behind other medical specialties in the UK. A 1997 survey of UK Palliative Medicine trainees identified they were highly motivated and interested in research. However, specialty trainees (StRs) have reported variable access to research opportunities by region of practice. This survey aimed to explore research interest, opportunities and barriers to research for UK StRs.
Methods Online Surveys software was used to design two surveys; one for Training Programme Directors (TPDs) \& one for StRs. The Association of Palliative Medicine Science Committee (SAC) reviewed both surveys and then emailed the survey link to all UK TPDs with a request to cascade the StR survey within their region.

Results TPDs responded from 14/16 (88\%) postgraduate training regions. 102/225 (45\%) of UK StRs responded, representing all training regions. $13(6 \%)$ of StR participants reported having research time through clinical academic/ research fellow posts or time out of training. 92\% (94/102) of StRs reported being interested in research with 20\% (22/ 102) completing a postgraduate qualification including research. The main barriers identified were limited research supervision and time. Although 49\% $(\mathrm{n}=50)$ of StRs reported having access to a supervisor supportive of research, only $34 \%(n=35)$ stated they had access to a supervisor with research experience. The amount of research time varied greatly, from StRs reported no dedicated research time $(60 \%$, $\mathrm{n}=61$ ) up to a maximum of 8 months during training. Although the majority had opportunities to access journal clubs $(76 \%, \mathrm{n}=77)$ or research meetings $(56 \%, \mathrm{n}=57)$ many trainees $(42 \%, n=42)$ still reported mainly doing research in their own time.

Conclusion Palliative care delivers complex questions that warrant quality research. This survey identifies variability in adequate support and opportunities for StRs to facilitate research. Improving research capability and capacity in training is therefore imperative.

\section{NOVEL USE OF HIGH-FIDELITY SIMULATION IN THE TRAINING OF PALLIATIVE CARE HOSPITAL LIAISON TEAM NURSES IN THE MANAGEMENT OF PALLIATIVE CARE EMERGENCIES}

Rowan Walmsley, Deepta Churm, Leonie Armstrong, Nichola Jenkins, Sharon Hartley, John Stratford. Northumbria Healthcare NHS Foundation Trust

\subsection{6/spcare-2020-PCC.63}

Introduction Northumbria Healthcare has a well-established hospital liaison palliative care team (HLT), working over 4 different hospital sites. Comprising mostly of palliative care nurses, they see patients throughout their hospital admissions, sometimes in emergency situations. The need to improve skills in this area was recognised, so we piloted a novel education package using simulation.

Methods We delivered 3 half days of simulation training to 9 HLT palliative care nurses ranging from band 5 to 7 in seniority. The scenarios represented several emergency topics, including massive haemorrhage, seizures, hypercalcaemia, and superior vena cava obstruction. We used high-fidelity simulation manikins, with members of the simulation faculty roleplaying healthcare professionals or relatives. The scenarios ran for 20 minutes followed by a 30 minute debrief. Evaluation forms were completed at the end, where participants rated their confidence levels before and after completing the scenario, how realistic the scenarios were, how helpful the debrief was, and whether they would recommend this style of teaching to their colleagues. They also gave free text comments.

Results Most of the participants rated their confidence higher following the simulation than before it. Six participants agreed 